\title{
Hybrid Code Development for the Numerical Simulation of Instationary Magnetoplasmadynamic Thrusters
}

\author{
M. Fertig ${ }^{1}$, D. Petkow ${ }^{1}$, T. Stindl ${ }^{1}$, M. Auweter-Kurtz ${ }^{2}$, M. Quandt ${ }^{3}$, \\ C.-D. Munz ${ }^{3}$, J. Neudorfer ${ }^{4}$, S. Roller $^{4}$, D. D'Andrea ${ }^{5}$, and R. Schneider ${ }^{5}$ \\ 1 Institut für Raumfahrtsysteme, Abt. Raumtransporttechnologie, Universität \\ Stuttgart, Germany fertig@irs.uni-stuttgart.de \\ 2 Universität Hamburg, Germany Praesidentin@HVN.uni-hamburg.de \\ 3 Institut für Aerodynamik und Gasdynamik, Universität Stuttgart, Germany \\ munz@iag.uni-stuttgart.de \\ ${ }^{4}$ Höchstleistungsrechenzentrum Stuttgart, Stuttgart, Germany roller@hlrs.de \\ 5 Forschungszentrum Karlsruhe, Institut für Hochleistungsimpuls- und \\ Mikrowellentechnik, Germany rudolf.schneider@ihm.fzk.de
}

Summary. This paper describes the numerical modeling of rarefied plasma flows under conditions where continuum assumptions fail. We numerically solve the Boltzmann equation for rarefied, non-continuum plasma flows, making use of well known approaches as PIC (Particle in Cell) and as DSMC (Direct Simulation Monte Carlo). The mathematical and numerical modeling is explained in some detail and the required computational resources are investigated.

\section{Introduction}

Within the small satellite program of the Institute of Space Systems at University of Stuttgart a lunar satellite is under development. The main propulsion system will consist of a cluster of instationary magnetoplasmadynamic (IMPD) thruster, also known as pulsed plasma thruster. The duration of a single pulse of the IMPD thruster is of the order of $8 \mu \mathrm{s}$. The current of about 30 $\mathrm{kA}$ allows an acceleration of the propellant mass bit leading to exhaust veloci- 
ties of about $12 \mathrm{~km} / \mathrm{s}$, i.e. a specific impulse of approximately $1200 \mathrm{~s}$ [13]. Due to the instationary operation and the degree of rarefaction, discontinuities in the space distribution of the propelling plasma are to be expected. In order to significantly enhance the understanding of such an electric space propulsion system a realistic simulation of the observed rarefied plasma flow is needed. In this paper we describe the development of such a plasma flow simulation tool for complex three-dimensional geometries. The circumstance of rarefied plasma flow requires a kinetic description: The interaction of charged particles with electromagnetic fields requires the solution of the time-dependent full Maxwell-Vlasov system in three space dimensions for complex geometries. An attractive numerical method for this system is the Particle-in-Cell (PIC) approach $[2,8]$. Unfortunately, this method does not take into account elastic and inelastic scattering between particles. However, since these interactions can play an important role for the thrust of electric propulsion systems, it is necessary to include them. In our approach the exchange of momentum and energy as well as chemical reactions is calculated via a Direct Simulation Monte Carlo (DSMC) method [1, 12]. A new developed Fokker-Planck solver [5], also using PIC techniques in velocity space, numerically models electron-electron and electron-ion Coulomb collisions in a self-consistent way. It is expected that the coupling of these models, i.e. PIC-DSMC-FP, allow for an accurate prediction of the thrust of electric space propulsion systems operating far from continuum.

In general, the coupling of different particle approaches for the numerical investigations of rarefied flows in the field of electric propulsion is a well known proceeding. In case of Hall thrusters it is common to treat the electrons as a fluid and the atoms and ions as particles, see e.g. [11, 4]. A rise in computational power allowed to apply fully kinetic (PIC-DSMC) approaches [10]. Most of the available Hall thruster studies are based on additional simplifying assumption concerning the neutral particle distribution, see e.g. [6]. In the field of pulsed plasma thrusters (PPT), much less particle based studies are available, see e.g. [7, 3, 9]. However, these models cannot resolve the question about how strong the deviation of the Maxwellian electron distribution is due to high gradients, unknown collisionality and electron transport. 


\section{References}

1. G.A. Bird. Molecular Gas Dynamics and the Direct Simulation of Gas Flows. Clarendon Press, Oxford, 1994.

2. C.K. Birdsall and A.B. Langdon. Plasma Physics via Computer Simulation. Adam Hilger, Bristol, Philadelphia, New York, 1991.

3. I. D. Boyd, M. Keidar, and W. McKeon. Modeling of a pulsed plasma thruster from plasma generation to plume far field. Journal of Propulsion and Power, 37(3):399-407, 2000.

4. S. Cheng, M. Santi, M. Celik, M. Martinez-Sanchez, and J. Peraire. Hybrid pic-dsmc simulation of a hall thruster plume on unstructured grids. Computer Physics Communications, 164:73-79, 2004.

5. D. D'Andrea, C.-D. Munz, and R. Schneider. Modeling of electron-electron collisions for particle-in-cell simulations. FZKA 7218 Research Report, Forschungszentrum Karlsruhe - in der Helmholtz-Gemeinschaft, 2006.

6. L. Garrigues, A. Heron, J. C. Adam, and J. P. Boeuf. Hybrid and particlein-cell models of a stationary plasma thrusters. Plasma Sources Sci. Technol., 9:219-226, 2000.

7. N. A. Gatsonis and X. Yin. Hybrid (particle-fluid) modeling of pulsed plasma thruster plumes. Journal of Propulsion and Power, Vol. 17, No. 5, September-October 2001, 945-958, 2001.

8. G.B. Jacobs and J.S. Hesthaven. High-order nodal discontinuous Galerkin particle-in-cell method on unstructured grids. J. Comput. Phys., 214:96 - 121, 2006.

9. M. Keidar, I. D. Boyd, E. Antonsen, and G. G. Spanjers. Electromagnetic effects in the near-field plume exhaust of a micro-pulsed-plasma thruster. Journal of Propulsion and Power, 20(6):961-969, November 2004.

10. K. Komurasaki, S. Yokota, S. Yasui, and Y. Arakawa. Particle simulation of plasma dynamics inside an anode-layer hall thruster. 40th AIAA/ASME/SAE/ASEE Joint Propulsion Conference and Exhibit, 11-14 July, Fort Lauderdale, Florida, USA, 2004.

11. J. W. Koo and I. D. Boyd. Computational model of a hall thruster. Computer Physics Communications, 164:442-447, 2007.

12. K. Nanbu, T. Morimoto, and M. Suetani. Direct simulation monte carlo analysis of flows and etch rate in an inductively coupled plasma reactor. IEEE Trans. Pl. Sc., 27(5):1379-1388, 1999.

13. A. Nawaz, M. Auweter-Kurtz, G. Herdrich, and H. Kurtz. Investigation and optimization of an instationary MPD thruster at IRS. International Electric Propulsion Conference, Princeton, USA, 2005. 